\title{
La señal electrodérmica mediante Sociograph: metodología para medir la actividad grupal
}

\author{
Montserrat Aiger, María Palacín y José-Manuel \\ CORNEJO \\ Universidad de Barcelona
}<smiles>c1ccccc1</smiles>

\section{Resumen}

Se presenta una propuesta metodológica desde la perspectiva de la Neurociencia Social, utilizando la actividad electrodérmica (EDA) como marcador somático con el objetivo de medir la conducta grupal. Sociograph es un instrumento que permite innovar tecnológicamente y al ser poco intrusivo, permite investigar y medir el comportamiento atencional y emocional del grupo durante su actividad. El modelo biopsicosocial, base de las Neurociencias, exige instrumentación metodológica adecuada para su conceptualización y análisis. Sociograph permite registrar el nivel de activación atencional $(E D L g)$ y emocional $(E D R g)$ a través de un indicador somático (EDA), aplicado a la actividad grupal (EDAg). Sociograph hace posible realizar un seguimiento continuo en tiempo real y en formato digitalizado del registro psicofisologico de la actividad grupal durante la interacción, la interdependencia e intercambio de información en el grupo. Se ba creado una metodología de registro psicofisiológica de la señal grupal (EDAg) secuenciada en "Módulos de actividad" de medidas continuas en el tiempo. Cada estímulo que se presenta al grupo se segmenta para el análisis en secuencias modulares y da lugar a un determinado modelo secuencial que corresponde a una situación experimental pre-determinada. El registro grupal (EDAg) incorpora software para tratar y analizar la señal electrodérmica grupal. Estas aplicaciones son ELECTRO, VECTRO y VECTRA, creadas ad hoc para el análisis de señales electrofisiológicas grupales. A modo de ejemplo se aplica la metodología de registro psicofisiológico en la medición de la actividad "Tangram" en situación de co-presencia (sin interacción ni interdependencia) versus actividad grupal (con interacción e interdependencia). Se registran un total de 18 grupos con 5 personas en cada grupo. Los resultados ilustran la existencia de un patrón de activación psicofisiológico propio de la actividad grupal y la metodología utilizada permite verificar que el grupo reacciona de manera diferente a contextos estimulares distintos. La EDAg resulta un buen indicador somatico para evaluar el comportamiento psicosocial, y resulta apropiado para el estudio del comportamiento grupal, en su nivel de análisis intragrupal.

Palabras clave: Actividad Electrodérmica grupal (EDAg), actividad grupal, metodología y neurociencia social.

\section{Electrodermal signal using Sociograph: Methodology to measure group activity}

\begin{abstract}
A Social Neuroscience approach is adopted to present a methodology proposal using electrodermal activity (EDA) as a somatic marker in order to measure group behaviour. Sociograph is an unobtrusive instrument providing technological innovation, which allows researchers to study and measure the group's attentional and emotional behaviour during its activity. The biopsychosocial model, basis of neuroscience, requires adequate methodological instrumentation for its conceptualization and analysis. Sociograph allows the recording of attentional $(E D L g)$ and emotional $(E D R g)$ levels of activation through a somatic indicator (EDA), applied to the group activity (EDAg). With Sociograph it is possible to carry out continuous real-time and digitized format monitoring of psychophysiological registry of group activity during the interaction, interdependence and exchange of information in the group. We have developed a methodology for recording psychophysiological group signal (EDAg), sequenced in 'Activity modules' of continuous measures over time. Each stimulus presented to the group is segmented for analysis in modular sequences, and results in a specific sequential pattern corresponding to a predetermined experimental situation. The group registration (EDAg) incorporates software to process and analyze the electrodermal group signal. These applications are ELECTRO, VECTRO and VECTRA, created ad boc for electrophysiological group signal analysis. As an example, we apply psychophysiological recording methodology in the measurement of the "Tangram" activity in a situation of copresence (without interaction or interdependence) versus group activity (with interaction and interdependence). A total of 18 groups with 5 participants in each group were recorded. The results illustrate the existence of a psychophysiological activation pattern typical of group activity, and the methodology used allows us to verify that the group reacts differently to different stimulus contexts. The EDAg is a good somatic indicator for measuring psychosocial behaviour, and it is appropriate for studying group behaviour in in-group level of analysis.

Keywords: Electrodermal Group Activity (EDAg), group activity, methodology and social neuroscience. Correspondencia con los autores: Montserrat Aiger. Departamento de Psicología Social, Facultad de Psicología,
Universidad de Barcelona, Paseo de la Vall d Hebrón 171, 08035 Barcelona. Tlf: 93312517 . Fax: 934021366. E-mail: montseaiger@ub.edu

Original recibido: 9 de enero de 2013. Aceptado: 14 de marzo de 2013.
\end{abstract}


Revista de Psicología Social, 2013, 28 (3), pp. 0-0

\section{Paradigmas teórico-metodológicos en la investigación grupal}

La preocupación por la metodología de investigación en la Psicología Social y de los Grupos se desarrolla y fluctúa por periodos que muestran preferencias cíclicas. En algunos momentos hay una preferencia por lo experimental y de laboratorio, en otros por lo cuasi-experimental y correlacional, y en otros por los estudios de campo y grupos naturales (Festinger y Katz, 2004).

Podríamos afirmar que existen tres grandes paradigmas teórico-metodológicos que han orientado la investigación dando lugar a tres modalidades de investigar lo social y por ende lo grupal que se pueden resumir de la siguiente forma:

- Una modalidad caracterizada por la investigación de grupos y/o relaciones interpersonales, de carácter presencial. El grupo en su conjunto está presente en la investigación, tanto desde finales del S. XIX y hasta los años 60 se enfatiza la investigación en la polaridad individuo-grupo y en la superioridad de una perspectiva sobre la otra. En la década de los 30-40, hay un incremento de la sofisticación metodológica, y en los años 50-60 la investigación se amplía a otras áreas de investigación, la organizacional, como hasta el momento lo había sido la clínica, pero siempre en formato presencial. Se requiere la presencia de los sujetos experimentales, que son objeto de investigación, para medir cualquier actividad grupal experimental. Los instrumentos utilizados han sido la observación sistemática (mediante protocolos de observación creados ad hoc, flujos de participación, etc.) también la observación participante, el análisis de contenido, encuestas sociales, escalas de actitudes, técnicas sociométricas, entre las más destacadas.

- Desde finales de la década de los 70, con la aparición del paradigma del grupo mínimo se genera una nueva perspectiva a la investigación de corte sociocognitivista que dota de nuevas fórmulas experimentales para investigar lo grupal (y también lo intergrupal), distintas a las que hasta ese momento habían sido utilizadas. Esto implica para la Psicología Social Europea un auge en la investigación que se trasladará al mundo anglosajón. La investigación Social, especialmente la grupal, amplía escenarios y nuevos formatos para medir el efecto del grupo sobre el individuo. Las relaciones intergrupales toman relevancia y la metodología de registro sigue siendo de encuestas, escalas y observación directa, pero incluye otros modos que la tecnología favorece, como son: fotografía, ordenador y grabación audiovisual, entre los más destacados.

- El final del pasado siglo veinte y la primera década del siglo veintiuno son testigos del nacimiento de las Neurociencias y del desarrollo de la instrumentación técnica y metodológica para el estudio de la conducta social. El modelo biopsicosocial facilita el idear y aplicar instrumentación que permita medir dicha interrelación desde lo empírico. Por ejemplo, con la implementación de las técnicas de neuroimagen funcional como son: la Tomografía por emisión de positrones (PET), Resonancia magnética funcional (FMRI), Potenciales relacionados con eventos (ERP), Tomografía computarizada por emisión de fotón único (SPECT), Magnetoencefalografía (MEG), Neuroimagen multimodal y técnicas de registro electrofisiológicas (EDA) (Alcover, 2008; Harmon-Jones y Beer, 2009). La evolución de la instrumentación psicofisiológica y la innovación técnica de la neuroimagen han servido para el desarrollo de esta nueva perspectiva teórico-metodológica actual.

\section{La instrumentación psicofisiológica}

El auge de la Psicofisiología Social surge a partir de la década de los 60 (Schachter y Singer, 1962) en torno a los determinantes socio-cognitivos de los estados emocionales, el arousal y la co-presencia (Zajonc, 1965), y la 
medición de las actitudes (Cacioppo, 1982). En la sistematización de esta área contribuye la publicación en 1983 de "Social psychophysiology: A sourcebook", editado por Cacioppo y Petty. Y en la actualidad, el estudio de las Neurociencias Sociales, con el uso de instrumentación de neuroimagen funcional, ha permitido su desarrollo. Como ejemplo de esto último, están los marcadores somáticos en investigaciones multidisciplinares (Damasio, 1998, 2005).

Las técnicas psicofisiológicas más utilizadas en Psicología Social son: Electrocardiología (mide tasa cardíaca), la Electromiografía (mide los diferentes patrones de contracción de la musculatura facial) (Sirota y Schwartz, 1982) y la relación con las emociones, estados de ánimo, respuesta sexual y actitudes (Cacioppo y Petty, 1983; Cacioppo, Petty y Marshall-Godell, 1984; McHugo, Lanzatta, Sullivan, Masters y Englis, 1985). La Electroencefalografía (EEG) centra sus registros en el sistema nervioso central (SNC). Otros se han centrado en la asimetría hemisférica y las emociones. Las técnicas de registro de potenciales bioeléctricos directos, la electro-oculografía, como respuesta ocular a situaciones de interacción (Young y Sheena, 1975), y los registros de voz que permiten discriminar patrones acústicos característicos de cada emoción (Muñoz y Jiménez, 1990). El estudio de la actividad electrodérmica y la tasa cardiaca (Corral, Otero, Barrenetxea y Landeta, 1998) que permite establecer patrones de respuesta vinculados a perfiles de personalidad (Jacobs y Schucker, 1981), y como reacción a situaciones de interacción.

\section{La actividad electrodérmica en la medición psicosocial}

La instrumentación psicofisiológica de la actividad electrodermal (EDA), ampliamente utilizada en investigación de procesos psicológicos, se ha centrado en medir procesos intrapersonales, actitudes y estados del arousal. La sensibilidad, fiabilidad y facilidad de medición (Martínez, Garrido y Valdunquillo, 2005) permiten que se convierta en una de las principales técnicas actuales de medición de fenómenos psicosociales. En la medición de actitudes, el registro de la EDA más utilizado ha sido la resistencia de la piel (Shapiro y Schwartz, 1970). La EDA se convierte en un índice psicofisiológico útil por su relación con la emoción, el arousal y la atención (Dawson, Schell y Filion, 2007; Lajante, Dondainet y Amarantini, 2012; MarshallGoodell, Tassinary y Cacioppo, 1990; Micu y Plummer, 2010). Sus variaciones se entienden como una evidencia de cambios en el estado cognitivoemocional de la persona (Hugdahl, 1995; McHugo y Lanzetta, 1983; Moya y Salvador, 2001), y constituye un marcador adecuado para la medición de alerta cortical y emocional (Bechara, Damasio, Tranel y Damasio, 2005; Damasio, 1998, 2005; Harmon-Jones y Beer, 2009; Harmon-Jones y Winkielman, 2007; Martínez, Sánchez, Bechara y Román, 2006; Sequeira, Hot, Silvert y Delplanque, 2009).

La EDA se define como el cambio en las propiedades eléctricas de la piel en respuesta a la secreción de sudor por las glándulas sudoríparas ecrinas. Estas glándulas se encuentran principalmente en la dermis de la zona palmar y plantar y están bajo el control del sistema nervioso autónomo (SNA) cuyas órdenes se transmiten para activar el sistema nervioso central (SNC) (Sequeira et al., 2009). Boucsein $(1992,2012)$ destaca tres subsistemas de control de EDA: (a) estructuras límbicas (hipotálamo, corteza cingulada giro e hipocampo) implicados en las respuestas emocionales y la termorregulación, (b) la corteza motora y los ganglios basales implicados en la locomoción y (c) la formación reticular involucrada en el control del nivel de excitación. En la actualidad hay plena aceptación den- 
tro de la comunidad científica de que las variaciones en las propiedades eléctricas de la piel son marcadores de eventos relevantes en el campo de las emociones y la atención (Dawson, Schell y Courtney, 2011; Sequeira et al., 2009).

En la EDA se diferencian tres componentes básicos: el nivel (N), la respuesta (R) y la actividad espontánea. El nivel se refiere al registro tónico, con las siglas EDL, y el registro fásico, con las siglas EDR. La actividad espontánea se refiere a la actividad psicofisiológica no específica (NSA) (Benedek y Kaernbach, 2010; Boucsein, 1992, 2012).

\section{Registro electrodérmico para medir la actividad grupal: Sociograph}

Sociograph es una innovación técnica que proviene de la Ingeniería electrónica, y surge originalmente para medir las reacciones colectivas (Martínez y Garrido, 2003).

Sociograph es un instrumento electrónico que registra la actividad electrodérmica (mediante la resistencia dérmica) de un grupo en una determinada actividad grupal (EDAg) en los niveles tónicos y fásicos (EDLg - EDRg), a través del método de corriente constante expresa sus valores en Kilohmios, permite la evaluación del nivel de activación cortical y respuestas emocionales mientras realizan dicha actividad. Sociograph sigue el principio del método exosomático de registro de la actividad electrodérmica, tan ampliamente admitido (Dawson et al., 2007; Ramos, 2006), mediante el que se miden los cambios en la piel al paso de una pequeña corriente eléctrica aplicada externamente. Dicho método permite registrar la activación de los sujetos experimentales, como grupo, a través de un parámetro (EDAg). Es decir, la respuesta grupal atencional y emocional se integra durante la realización de la actividad experimental.

Sociograph, como instrumento técnico es útil en Psicología de los Grupos, ya que implica registrar la reacción de un grupo de sujetos ante diferentes estímulos mientras realizan una tarea determinada, durante la que se mide el nivel de activación cognitiva-emocional.

Sociograph registra el nivel de activación cortical y la respuesta emocional que generan los miembros de un grupo a través del indicador somático de la actividad grupal (EDAg), que se toma como unidad de análisis de la respuesta integrada de ese conjunto de sujetos (en la pueden evaluarse hasta 16 sujetos). A través del parámetro electrofisiológico (EDAg) se registra la actividad durante el tiempo en que se produce la interacción e interdependencia para conseguir el objetivo grupal propuesto. Por tanto la actividad grupal se puede medir y calibrar con cierta precisión cuantificada.

Sociograph está compuesto por dos unidades técnicas (ver Figuras 1 y 2). La primera, los bioamplificadores, con dos salidas, una para la actividad electrodérmica tónica (EDL) y otra para la actividad electrodérmica fásica (EDR). La medición de la EDAg se realiza a través de dos electrodos de acero inoxidable que se sitúan en la segunda falange de los dedos anular e índice. Esta señal (EDAg) es continua y permite realizar un seguimiento de la actividad del grupo en tiempo real. Y la segunda es Sociolab, que procesa el registro de la información del grupo y está constituida por: Group Response (GR) que procesa de forma conjunta las señales individuales (EDR) para establecer las respuestas del grupo (EDRg), y Group Activity (GA) que registra el nivel de la activación del grupo (EDLg).

Ambas señales grupales están en la salida del equipo (Sociograph) para ser procesadas a través del convertidor analógico-digital (PowerLab 4/26 Channel Recorder). Éste permite la visualización y análisis de la señal grupal mediante la aplicación del software Powerlab. Posteriormente, para analizar de manera específica la señal grupal (EDAg) se utiliza la aplicación informática ELECTRO (Cornejo, 
2008). Este Software trata las señales electrofisiológicas grupales mediante un tratamiento estadístico matemático que condensa la señal. Ésta se aglutina en unidades de tiempo y reciben un posterior tratamiento de medias por bloques significativos y análisis de secuencias temporales, ya que la señal es muy sensible a multitud de indicios e incidentes que se procesan en cada milésima de segundo.

Para una visualización rápida de la señal, $\operatorname{VECTRO~(Cornejo,~2011a),~permite~}$ analizar información complementaria sobre los perfiles de relación entre la EDLg-EDRg y VECTRA (Cornejo, 2011b), permite un análisis en profundidad sobre cada uno los Módulos de actividad.

FIGURA 1

Instrumentación para el registro de la Actividad Electrodérmica en los grupos (EDAg)

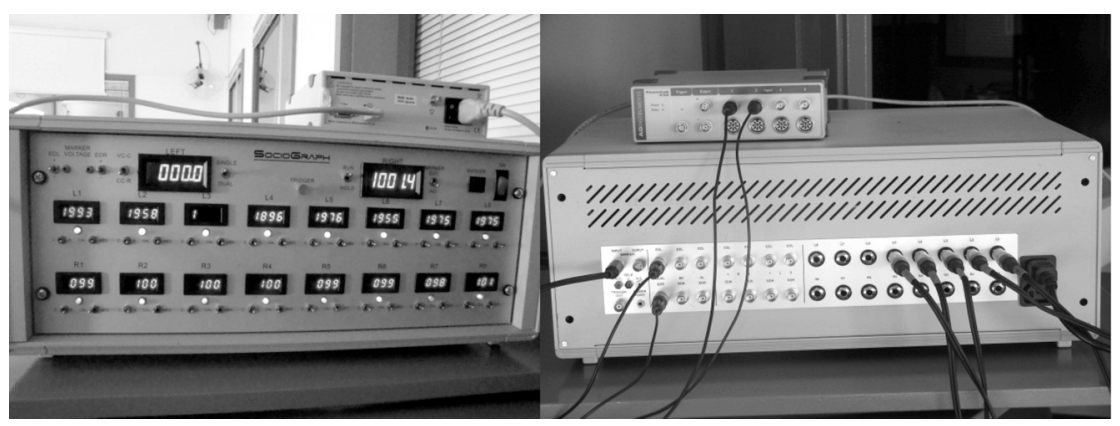

Parte frontal de Sociograph

B. Parte posterior de Sociograph y conexión con el PowerLab

FIGURA 2

Diagrama de bloques y modelización matemática de Sociograph

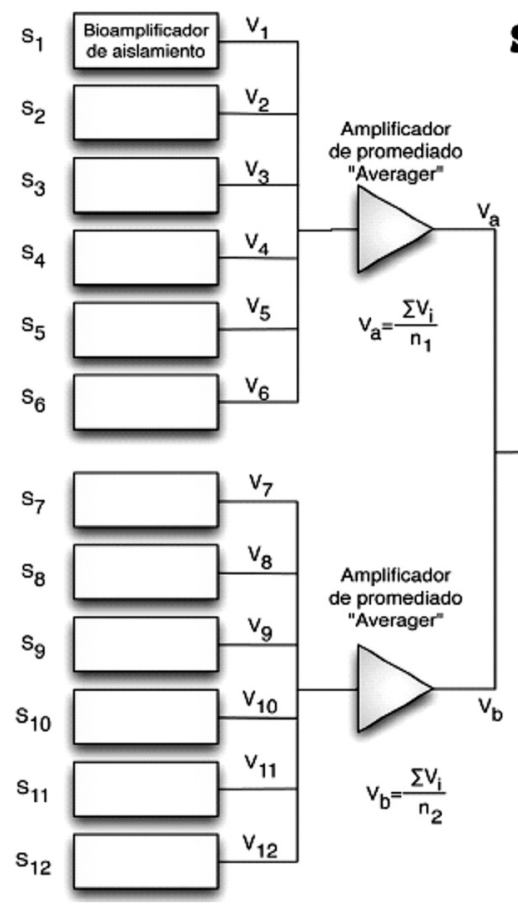

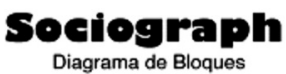


Actividad Fásica

Fuente variable de tensión de referencia $\left(V_{R}\right)$ con el fin de igualarlo con $V_{1}$ y amplificar las diferencias de tensión 
6 Revista de Psicología Social, 2013, 28 (3), pp. 0-0

\section{Metodología específica de registro de la señal grupal: Diseño de estructura modular}

El registro de la actividad grupal sociográfica, mediante la EDAg de los miembros del grupo, permite realizar el seguimiento del grupo en tiempo real (medidas continuas), como se ha comentado con anterioridad, ante la influencia del estímulo social.

Para el registro de la EDAg se segmenta la actividad experimental en espacios de actividad diferenciados que implican tiempos de secuencia específicos que permitirán un análisis minucioso de la señal. Estas secuencias estructuradas son necesarias y permiten segmentando la señal, como variable de control, ver el efecto estimular del grupo según su actividad.

La creación de los Módulos de Actividad también implica:

- Incorporar una estructura modular para segmentar la medición de la secuencia natural de la evolución de una sesión de trabajo grupal a través de las fases de inicio, desarrollo y cierre, tal y como funciona un grupo en su actividad evolutiva normal (ver Figura 3).

- Crear franjas temporales en el registro grupal con la finalidad de estabilizar la señal (línea base) para que las oscilaciones tónicas y fásicas de la EDA, durante el registro de los Módulos de actividad, se deban a la reacción estimular concreta que se pretende medir y no a la actividad espontánea. Este elemento persigue garantizar la variancia del registro.

- Estructurar la evolución de la actividad grupal para dotar de constancia el orden en el que se dan las acciones grupales. La metodología de registro a través de las Secuencias modulares garantiza que todos los grupos de la muestra realizan los mismos Módulos en el mismo orden y en el mismo tiempo. Las Secuencias modulares permiten comparar Módulos de actividad a nivel intra-intergrupal, tanto en medidas repetidas e independientes, siguiendo una estrategia de análisis sincrónica y/o diacrónica.

FIGURA 3

Diseño de estructuras modulares para el registro de la EDAg

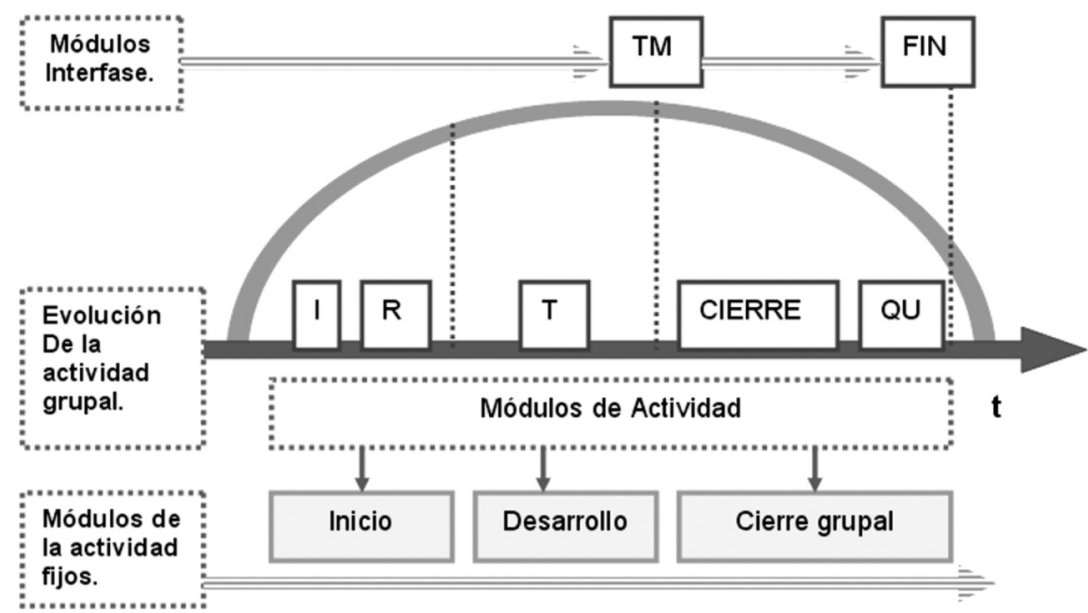

Nota: Las estructuras modulares se distribuyen según la evolución temporal de la actividad del grupo para segmentar el registro de la EDAg como señal continua durante el tiempo real en que se desarrolla la actividad experimental en el grupo.

- La creación de Módulos de actividad asociados a contextos estimulares (Freixa-Baqué, 2001) específicos, dentro de una misma secuencia temporal, son 
necesarios y útiles para facilitar el análisis posterior de la señal a través de los índices de la EDA. Los contextos estimulares aplicados a la actividad grupal son cuatro (ver Figura 4):

- Periodo de habituación a la situación experimental. Este contexto estimular permite destinar un tiempo a estabilizar la señal (línea base del grupo) antes de medir la tarea. Se han distribuido dos Módulos de actividad en él: Las instrucciones $(\mathrm{I})$ y la respiración $(\mathrm{R})$.

- Periodo de reposo durante el cual el grupo no recibe ningún estímulo externo objetivable o programado en el diseño. Este contexto estimular pertenece a los Módulos Interfase: Tiempo Muerto (TM) y Fin.

- Periodo de la fase estimular donde el grupo recibe estimulación continua, controlada y programada. Aquí se sitúa la tarea grupal (T).

- El periodo de estimulación discreta se refiriere al momento preciso que sigue a la presentación de un estímulo puntual. En el diseño de la Secuencia modular los Módulos de actividad que pertenecen a este contexto estimular tienen doble uso: como períodos de reposo y como períodos de estimulación discreta. Se resalta un perfil u otro según la intención de análisis del diseño. Pertenecen a esta tipología: el Tiempo Muerto y el Fin.

\section{FIGURA 4}

Distribución de los contextos estimulares de la señal grupal (EDAg) asociados a la Secuencia Modular de una sesión grupal



Nota: Se trata de la ubicación de los Módulos de actividad según su adecuación a la evolución y secuencia de la actividad grupal, en función de las características eléctricas que implica la EDA en su ciclo de registro.

En la figura 5 se describe la Secuencia modular de registro constituida por los Módulos de actividad y Módulos interfase. Los Módulos de actividad son franjas temporales en las que se da la actividad grupal. A continuación se describe la Secuencia modular al completo:

- Instrucciones (I): módulo destinado a dar las instrucciones asociadas a la actividad por el investigador. 
- Respiración $(R)$ : módulo destinado a la relajación previa al inicio de la tarea. Se trata de una condición necesaria para estabilizar la señal del grupo (línea base).

- Tarea (T): módulo destinado a la propia actividad grupal. Se indica con números (T1, y T2) cuando en una misma sesión de registro hay más de una actividad.

- Cierre $(C)$ : módulo destinado a la valoración de la actividad grupal.

- Cuestionario $(Q U)$ : módulo destinado a marcar el tiempo destinado a que los participantes del grupo contesten los cuestionarios de evaluación.

Los Módulos interfase son Módulos de actividad en los que el grupo no debe realizar una tarea propiamente, se trata de actividad espontánea del grupo, es un tiempo de espera o de tránsito entre módulos que permite controlar la actividad del grupo (bien dentro de un Módulo fijo de actividad o entre ellos). Los Módulos interfase son un registro estratégico para ver la respuesta del grupo ante una tarea informal o relacional, sin control por parte del investigador, quien guía las actividades. Hay dos tipologías (ver Figura 5):

- Tiempo Muerto (TM): Módulo que identifica el tiempo en el que el grupo espera para realizar otra actividad. El investigador no da instrucciones, ya que se trata de un tiempo de actividad informal.

- Fin de la actividad (FIN): Módulo que identifica el tiempo en el que el grupo ha finalizado su tarea experimental y se produce el cierre a continuación. El FIN implica el tiempo que trascurre después de responder a los instrumentos de evaluación hasta que ha finalizado el registro y se les saca los electrodos a los sujetos experimentales.

Los Módulos de actividad se agrupan en Módulos fijos de actividad, constituyendo Secuencias modulares de registro según la descripción de las variables independientes del diseño (ver Figura 5).

A modo de ejemplo se aplica esta metodología de registro (Secuencia modular) durante el registro de una actividad que realiza el grupo, a través de la tarea de "Tangram".

FIGURA 5

Modelo de la Secuencia modular para el registro de la actividad grupal



Nota: Este Modelo refleja la secuencialidad temática y temporal que siguen los grupos durante la realización de la tarea experimental. El objetivo es delimitar y segmentar el contexto estimular (Módulos de actividad) para explorar la reactividad (cognitiva-emocional) del grupo, en un determinado tiempo de la secuencia continua del registro de la EDAg durante la actividad del grupo.

\section{Método}

El registro de medida psicofisiológica de la actividad grupal se realiza en el Laboratorio de Psicología Social del Departamento de Psicología Social de la Facultad de Psicología de la Universidad de Barcelona. Se sigue la norma- 
tiva propia del código deontológico para la experimentación social que rige el reglamento de uso del Laboratorio de Psicología Social. Se describe a continuación el experimento grupal durante la realización de la actividad "Tangram”.

\section{Muestra}

La muestra de este trabajo se realiza con estudiantes de la Facultad de Psicología de Grado, Licenciatura y de Master de la Universidad de Barcelona compuesta por 18 grupos experimentales con cinco sujetos en cada grupo, con un total de 445.710 registros.

Esta muestra se extrae de una más amplia compuesta de 211 grupos que implica 790 sujetos, realizando un cómputo total de 10 actividades. A modo de ejemplo sólo se describe la actividad "Tangram" realizada por los grupos experimentales.

\section{Procedimiento}

Las actividades experimentales siempre las guía el experimentador, autor de la investigación, con ayuda de personal auxiliar de laboratorio. Los experimentos (registro de los 211 grupos) han sido llevados a cabo durante los cursos académicos de 2007-08, 2008-09 y 2009-10, en los meses de octubre y noviembre para asegurar las mismas condiciones ambientales, y también por la misma persona como investigador de Laboratorio.

Se describe la metodología temporal y estructuro-espacial que realizan los grupos en el proceso de experimentación del Laboratorio para el registro de cualquiera de las actividades grupales mediante el registro de la EDA con Sociograph. La actividad "Tangram”, pertenece a la Tipología de McGrath (1984) en la clasificación de Solución de problema. Esta actividad implica construir una figura geométrica a partir de siete piezas. Para ello se dispone de una cuartilla con la imagen a reproducir (como modelo) y debe hacerse en un tiempo determinado y conocido por los sujetos.

- El protocolo de actuación que se realiza en el laboratorio consta de cuatro fases secuenciales y progresivas que consisten en: (a) Fase preparatoria donde se realiza la configuración del instrumental técnico (PowerLab, Sociograph) y adecuación del escenario para el registro de la actividad (temperatura ambiente, estructura espacial de mobiliario, sillas, etc.). (b) Fase de entrada y recepción de los sujetos experimentales, el investigador y personal auxiliar reciben a los sujetos experimentales leyendo las condiciones de experimentación en el Laboratorio. (c) Fase de realización de la actividad experimental siguiendo la Metodología del Diseño de Estructura Modular que realiza el grupo en una secuencia de tiempo fija y predeterminada (ver Figura 5): Instrucciones (I1), Respiración (R1), Tarea (T1), Tiempo muerto (TM), Instrucciones (I2), Respiración (R2), Tarea (T2), Cierre (CIERRE), Cuestionario (QU) y Fin (FIN).

El grupo para realizar la actividad de "Tangram" pasa por tres módulos fijos de actividad. El primer módulo fijo de actividad realiza la actividad en co-presencia que incluye (I1), (R1) y (T1) y el módulo interfase (TM). Le sigue el módulo fijo de actividad grupal donde la actividad de Tangram se realiza de nuevo pero ahora con interacción e interdependencia y constituida por (I2), (R2) y (T2). El último módulo fijo de actividad grupal implica el cierre de la actividad grupal, (CIERRE), (QU). La actividad experimental finaliza con el módulo interfase (FIN). 
Revista de Psicología Social, 2013, 28 (3), pp. 0-0

- Y finalmente, (d) Fase de cierre y salida de los sujetos experimentales, donde se recoge el material, se despide y agradece la participación.

\section{Resultados}

Se presentan los resultados ${ }^{1}$ del nivel de activación de la EDAg registrados a través de la Secuencia modular de la actividad de "Tangram", tanto en el contexto estimular con interacción cuya actividad necesita de la interdependencia (actividad grupal), como en el contexto estimular cuya actividad no ha necesitado ni interacción ni interdependencia (co-presencia). El objetivo es comprobar cómo la actividad grupal sin interdependencia ni interacción produce una señal diferente a la actividad llevada a cabo por el grupo en la que sí se necesita interacción e interdependencia para conseguir el objetivo. La actividad de Tangram se realiza en dos escenarios de actividad diferentes y consecutivos.

En la figura 6 se presentan los resultados (media y desviación típica) de la EDAg de los diferentes Módulos de actividad. Y en la tabla I se exponen las diferencias de media de los Módulos de actividad de la señal tónica (EDLg) y las de la fásica $(E D R g)$. Se aplica el estadístico $Z$ para comparar los Módulos en los diferentes contextos de interacción social durante la realización de la actividad de "Tangram". Hay dos niveles de comparación entre estos. El primero delimita los Módulos en los que no hay interacción ni interdependencia (Módulos con el número 1) con aquellos en los que sí la hay (Módulos con el número 2) en diferentes contextos estimulares de interacción: Instrucciones (I1-I2) y cuando se realiza la actividad de "Tangram" (T1-T2).

Ambas comparaciones (I1-I2 y T1-T2) son significativas y se observa cómo en el grupo de personas que realizan el Módulo I2 (descenso de los valores de la EDLg) focalizan más la atención en comparación con el Módulo I1. La respuesta emocional (EDRg) en el I2 está más contenida (menos amplitud) que en la I1 (ver Figura 6). Se repite la misma tendencia oscilatoria en el par T1-T2 en la señal tónica, donde durante el T2 el grupo de personas focalizan más la atención (descenso de la resistencia dérmica) que en el T1, donde se realiza la actividad sin interacción ni interdependencia. La respuesta emocional (EDRg) en la T2 tiene mayor amplitud que la T1, aunque es más homogénea.

Y un segundo nivel de comparación entre los Módulos vinculados a la actividad grupal (Módulos con el número 2), con interacción e interdependencia. Primero se compara la actividad formal del grupo, pautada en interacción e interdependencia (T2) con la valoración de la actividad del grupo; abierta (Cierre). En segundo lugar la actividad grupal a nivel sincrónico (T2) con la valoración de la actividad grupal realizada a nivel diacrónico (QU). Y finalmente la valoración de la actividad grupal realizada de manera sincrónica e intragrupal (Cierre) con la valoración de la actividad realizada de manera diacrónica e intrapersonal (QU). Todos estos Módulos de actividad, tanto en la EDLg como en la EDRg, tienen diferencias de medias significativas ( $p$ $<.01$ ) (ver Tabla I). El grupo consigue focalizar más la atención (EDLg) cuando realiza una actividad grupal abierta (Cierre) que cuando es pautada y formal con normas (T2), siendo más homogénea la activación en el T2 que en el cierre (ver Figura 6). Y la respuesta emocional (EDRg) es más amplia en el T2 que en el Cierre, aunque más homogénea en la T2. Y finalmente el par Cierre-QU, donde el grupo focaliza más la atención (EDLg) cuando piensa en la actividad que ha realizado en él, de manera diacrónica (QU) que cuando hay interacción de manera sincrónica (Cierre), siendo también esta 
activación más homogénea en el QU. La respuesta emocional (EDRg) presenta menos amplitud el QU que el en Cierre, siendo más homogénea en éste último.

FIGURA 6

Puntuaciones de la media (M) y desviación típica (DT) de la EDLg y la EDRg de la Secuencia Modular de registro de la actividad "Tangram"



Nota: Las puntuaciones del descenso de la media en la EDLg a partir del Módulo T2 indican la focalización de la atención en el grupo durante la actividad de "Tangram".

TABLA I

Comparación de medias entre los Módulos de la actividad de Tangram

\begin{tabular}{llll}
\hline $\begin{array}{c}\text { Actividad } \\
\text { grupal }\end{array}$ & $\begin{array}{c}\text { Comparación de } \\
\text { medias por } \\
\text { 'Módulos' }\end{array}$ & \multicolumn{2}{c}{ Estadístico $Z$} \\
\hline Tangram & Par: I1-I2 & $\begin{array}{l}\mathrm{ZL}=536.21 \\
\mathrm{ZR}=18.34\end{array}$ & $\begin{array}{l}P<.01 \\
\boldsymbol{P}>.01\end{array}$ \\
\cline { 2 - 4 } & Par: T1-T2 & $\mathrm{ZL}=135.86$ & $P<.01$ \\
& Par: T2-Cierre & ZL $=44.95$ & $P<.01$ \\
\cline { 2 - 4 } & Par: T2-QU & ZR $=47.99$ & $P<.01$ \\
& Par: Cierre-QU & ZR $=223.24$ & $P<.01$ \\
& & ZL $=129.56$ & $P<.01$ \\
\cline { 2 - 4 } & ZR $=17.05$ & $P<.01$ \\
& & $P<.01$
\end{tabular}

Nota: En la comparación de medias a través del estadístico $Z$, la "L" es la sigla que designa la media de la señal tónica EDLg, y la "R" la media de la señal fásica EDRg.

Las puntuaciones máximas y mínimas absolutas de la actividad de "Tangram" marcan una amplitud de oscilación de la EDLg de 2.91 y en la EDRg de 14.53. En la figura 7 se presentan las puntuaciones máximas y mínimas promedio de los Módulos de actividad. En la tabla II constan los rangos de amplitud promedio de la señal tónica (EDLg) y fásica (EDRg) por Módulos de la actividad. 


\section{Revista de Psicología Social, 2013, 28 (3), pp. 0-0}

FIGURA 7

Promedio de las puntuaciones máximas - mínimas de la EDLg y la EDRg en la Secuencia Modular de registro de la actividad de "Tangram"

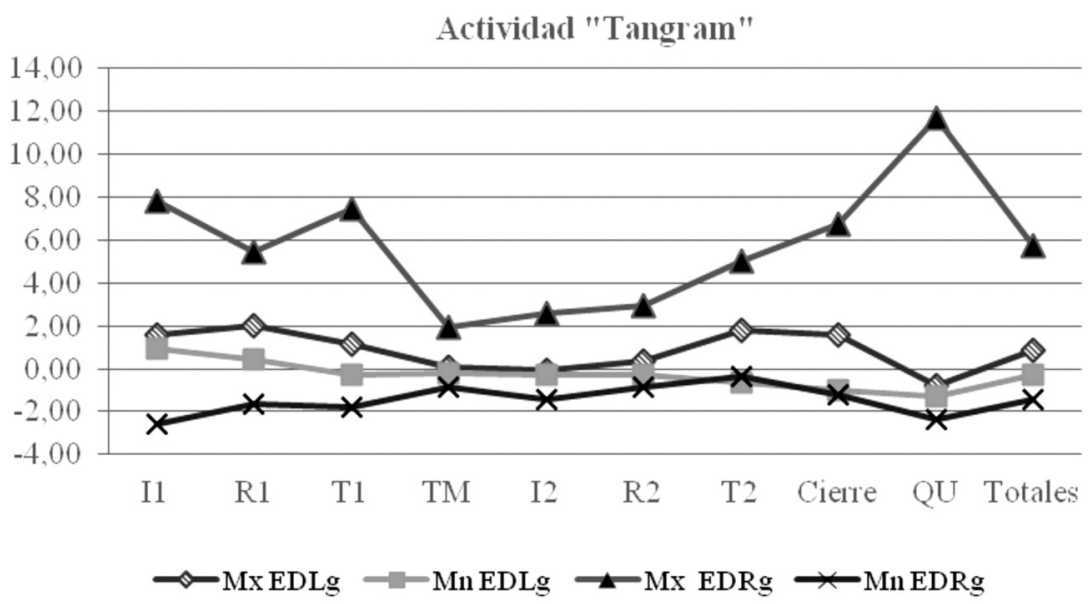

TABLA II

Media de los rangos de oscilación (puntuaciones máximas-mínimas) de la EDAg en los Módulos de la actividad grupal "Tangram"

\begin{tabular}{crr}
\hline $\begin{array}{c}\text { Módulos de } \\
\text { actividad }\end{array}$ & EDLg & EDRg \\
\hline I1 & 2.53 & 10.38 \\
R1 & 2.39 & 7.09 \\
T1 & 1.39 & 9.22 \\
TM & .32 & 2.73 \\
I2 & .27 & 3.99 \\
R2 & .61 & 3.76 \\
T2 & 2.48 & 5.34 \\
Cierre & 2.62 & 7.91 \\
QU & 2.09 & 14.03 \\
\hline
\end{tabular}

\section{DISCUSIÓN}

La metodología utilizada en el registro de la EDAg permite verificar que el grupo reacciona de manera diferente a contextos estimulares distintos. $\mathrm{La}$ metodología permite un registro sincrónico, en tiempo real y continuo mientras el grupo realiza su actividad. Ello resulta innovador y pionero con relación a las técnicas de registro en investigación grupal utilizadas hasta el momento actual. La estrategia metodológica de utilizar secuencias segmentadas, mediante los módulos de actividad, sirve para controlar el tiempo en que el grupo hace las tareas, y ello asegura el medir y visualizar la pauta de respuesta del grupo frente a los diferentes escenarios estimulares. La sistematicidad del procedimiento favorece y da consistencia al registro que se hace de la actividad grupal, lo que garantiza el control de las variables de estudio. Este es un requisito necesario en la investigación experimental, que siempre ha resultado difícil en la investigación básica de grupos. 
El diseño de secuencias modulares incorpora las variables de control de tres tipos: repetición, constancia y eliminación, garantizando así la sistematicidad y rigurosidad experimental requeridas.

Hasta la actualidad el registro de la EDA ha sido utilizado como técnica de registro individual, en este trabajo la medición a través de la EDA Grupal, permite obtener de forma segmentada registros del comportamiento atencional (EDLg) y emocional (EDRg) grupales.

La investigación desde las neurociencias ha ido desde el nivel molecular, celular, de sistemas, conductual y cognitiva tanto en lo intra personal como interpersonal. También el nivel intergrupal se ha incluido en el estudio, pero nunca el estrictamente intragrupal, objeto de estudio del presente trabajo. La innovación tecnológica ha permitido el estudio de los niveles citados, pero el nivel de explicación del comportamiento grupal, el intragrupal, carece de instrumentación tecnológica que permita su medición. La EDA medida con Sociograph permite el registro de los niveles de activación cognitivo y emocional del SNA (sistema nervioso autónomo), de forma poco intrusiva en los sujetos, y proporciona conocimiento de lo biológico, psicológico y social en el estudio del comportamiento grupal.

Se constata que los contextos estimulares donde el grupo realiza la actividad (T1) en co-presencia (sin interacción ni interdependencia) generan una activación atencional diferente a cuando realizan la actividad (T2) en grupo (con interacción e interdependencia). Esta tarea propiamente grupal, en la que hay interdependencia e interacción entre los sujetos, comporta un mayor nivel de activación (mayor descenso de la resistencia dérmica) que la realizada en grupo exclusivamente de co-presencia, sin interacción ni interdependencia. Cuando la actividad del grupo se realiza de manera conjunta, en interacción e interdependencia requiere más activación cognitiva, o focalización en la tarea por parte del grupo como unidad.

Otra conclusión de la señal grupal, es el incremento de la activación (EDLg) del grupo cuando piensa y evalúa la actividad grupal realizada (diacrónicamente) $(Q U)$ frente a cuando la está realizando de manera sincrónica (T2 y Cierre). La respuesta emocional (EDRg) en el grupo presenta su mayor contención (menor amplitud) cuando el grupo piensa en la actividad realizada $(Q U)$ frente a cuando éste interactúa para realizar dicha tarea (T2 y Cierre). El pensar en lo que se ha hecho en grupo genera mayor activación atencional (EDLg) y también mayor contención emocional (EDRg). La EDA se conceptualiza en la investigación experimental como un marcador (Bechara et al., 2005; Damasio, 1998, 2005; Martínez et al., 2006) y/o indicador somático adecuado para la medición de niveles de alerta cortical y emocional intra e interpersonal, y adecuados para la Psicología, las Ciencias Sociales y las situaciones de interacción social. Ello nos lleva a afirmar que la EDAg mediante el registro con Sociograph, también permite medir los niveles de activación grupales, en función de los escenarios estimulares del grupo. La EDAg resulta un buen indicador somático, constituye un parámetro óptimo para evaluar el comportamiento psicosocial, y resulta apropiado para el estudio del comportamiento grupal, en su nivel de análisis intragrupal.

Una de las limitaciones en la utilización de la EDAg es la alta sensibilidad y especificidad de respuesta de la señal que obliga a una necesaria delimitación del escenario de medición de las variables de estudio. El presente trabajo permite el estudio de la activación del grupo, como unidad de análisis, pero necesariamente para estudiar los procesos intra e intergrupales es necesario avanzar en las características morfológicas y de sintaxis de respuesta de la señal. 


\section{Revista de Psicología Social, 2013, 28 (3), pp. 0-0}

\section{Notas}

${ }^{1}$ El registro de la EDAg se realiza con el Sociograph y el PowerLab. La EDAg utiliza los Kilohmios (K $\Omega$ ) como unidad de medida de la resistencia dérmica en el tiempo. Se ha utilizado una cadencia de 20 registros por segundo. Se programa un rango de amplificación del canal 1 (EDLg) de $500 \mathrm{~K}$, y en el canal 2 (EDRg) de un rango de $10 \mathrm{~K} \Omega$, debido a sus diferencias morfológicas de activación (Boucsein, 1992, 2012).

\section{Referencias}

Alcover, C. M. (2008). Neurociencia social: hacia la integración de las explicaciones sociales y biológicas de la conducta social. En J. F. Morales, C. Huici, A. Gómez \& E. Gaviria (Coords.), Método, teoría e investigación en psicología social (pp. 187-214). Madrid: Pearson Prentice Hall

Bechara, A., Damasio, H., Tranel, D. \& Damasio, A. (2005). The Iowa gambling task and somatic marker hypothesis: Some questions and answers. Trends in Cognitive Sciences, 9, 159-162.

BenedeK, M. \& KaERnBACH, C. (2010). A continuous measure of phasic electrodermal activity. Journal of Neuroscience Methods, 190, 80-91. doi: 10.1016/j.jneumeth.2010.04.028

BoucseIN, W. (1992). Electrodermal activity. Nueva York: Plenum Press.

BoucseIn, W. (2012). Electrodermal Activity (2a ed.). Nueva York: Plenum Press.

Boucsein, W., Fowles, D. C., Grimnes, S., Ben-Shakhar, G., Roth, W. T., Dawson, M. E. \& Filion, D. L. (2012). Publication recommendations for electrodermal measurements. Psychophysiology, 49, 10171034.

CACiOppo, J. T. (1982). Social psychophysiology: A classic perspective and contemporary approach. Psychophysiology, 19, 241-251.

CACiopro, J. T. \& PetTy, R. E. (1983). Social psychophysiology: A sourcebook. Nueva York: Guildford Press.

Cacioppo, J. T., Petty, R. E. \& Marshall-Goodell, B. (1984). Electromyohraphic specificity during physical and actitudinal tasks. Biological Psychology, 19, 1-37.

CORnejo, J. M. (2008). Electro. Software de análisis de la señal eléctrica grupal (EDAg). Aplicación informática interna del Master Análisis y Conducción de Grupos del Departamento de Psicología Social. Universidad de Barcelona. Recuperado el 20 de octubre de 2008 de http://www.ub.edu/dppss/lps/lps_e.htm

CORnejo, J. M. (2011a). Vectro. Software de visualización de la señal electrodérmica grupal. Aplicación informática interna del Master Análisis y Conducción de Grupos del Departamento de Psicología Social. Barcelona: Universidad de Barcelona. Recuperado el 15 de mayo de 2011 de http://www.ub.edu/dppss/lps/lps_e.htm

Cornejo, J. M. (2011b). Vectra. Módulo de análisis de los bloques de actividad grupal. Aplicación informática interna del Master Análisis y Conducción de Grupos del Departamento de Psicología Social. Barcelona: Universidad de Barcelona. Recuperado el 15 de mayo de 2011 de http://www.ub.edu/dppss/lps/lps_e.htm

Corral, S., Otero, J., Barrenetxea, A. \& Landeta, O. (1998). Información y test de conocimiento culpable en la detección del engaño. Psicológica, 19, 187-199.

DAmAsio, A. (1998). El error de Descartes: emoción, razón y cerebro bumano. Barcelona: Crítica.

Damasio, A. (2005). En busca de Spinoza. Neurobiología de la emoción y los sentimientos. Barcelona: Crítica.

Dawson, M. E., SCHell, A. M. \& COURTNey, C. G. (2011). The skin conductance response, anticipation and decision-making. Journal of Neuroscience, Psychology, and Economics, 4, 111-116. doi:10.1037/a0022619

Dawson, M. E., Schell, A. M. \& Filion, D. L. (2007). The electrodermal system. En J. T. Cacioppo, L. G. Tassinary \& G. G. Bernston (Eds.), Handbook of psychophysiology (3a ed.) (pp. 159-181). Nueva York: Cambridge University.

Festinger, L. \& Katz. D. (Comps.) (2004). Los métodos de investigación en las ciencias sociales. Barcelona: Paidós Básica.

FreIXA I BAQUÉ, E. (2001). La actividad electrodérmica: historia, clasificación y técnicas de registro. Revista Internacional de Psicología Clinica y Salud, 1 (3), 529-545.

HARMON-JONES, E. \& BeER, J. S. (Eds.) (2009). Methods in social neuroscience. Nueva York: Guilford Press.

HARMON-JONES, E. \& Winkielman, P. (Eds.) (2007). Social neuroscience. Integrating biological and psychological explanations of social behavior. Nueva York: Guilford Press.

Hugdahl. K. (1995). Dichotic listening: Probing temporal lobe functional integrity. En R. J. Davidson \& K. Hugdahl (Eds.), Brain asymmetry (pp. 123-156). Cambrigde, MA: MIT Press.

JaCoBs, D. R. \& SCHUCKer, B. (1981). Type a behavior pattern, speech and CHS. En D. J. Darby (Ed.), Speech evaluation in medicine (pp. 210-217). Nueva York: Grune \& Stratton.

Lajante, M., Dondainet, T. \& Amarantini, G. (2012). Opening the 'black box' of electrodermal activity in consumer neuroscience research. Journal of Neuroscience, Psychology and Economics, 5, 238-248. doi: $10.1037 / \mathrm{a} 0030680$

Marshall-Goodell, B. S., Tassinary, L. G. \& Cacioppo, J. T. (1990). Principles of bioelectrical measurement. En J. T. Cacioppo \& L. G. Tassinary (Eds.), Principles of psychophysiology: Physical, social, and inferential elements (pp. 113-148). Nueva York: Cambridge University Press. 
MARTínez, J. L. \& GARRido, E. (2003). Sistema para la medición de reacciones emocionales en grupos sociales. 2168928 (Universidad de Salamanca). Patio de escuelas menores, nº1, 37007 Salamanca, España. Patente de invención. A6113 5116, 2003-10-1.

Martínez, J. L., Garrido, E. \& Valdunquillo, M. A. (2005). Desarrollo tecnológico para la medida electrofisiológica de la atención y activación emocional en grupos sociales-Sociograph-. En J. Romay \& R. García (Eds.), Aspectos históricos, teóricos y metodológicos. Madrid: Biblioteca Nueva.

martínez, J. M., Sánchez, J. P., Bechara, A. \& Román, F. (2006). Mecanismos cerebrales de la toma de decisiones. Revisión en Neurociencia, 42, 411-418.

McGrath, J. E. (1984). Groups. Interaction and performance. Englewood Cliffs, NJ: Prentice-Hall.

McHugo, G. J. \& LAnzetTa, J. T. (1983). Methodological decisions in social psychophysiology. En J. T. Cacioppo \& R. E. Petty (Eds.), Social psychophysiology: A sourcebook (pp. 630-665). Nueva York: Guilford.

Mchugo, G., Lanzetta, J. T., Sullivan, D. G., Masters, R. D. \& Englis, B. G. (1985). Emotional reactions to a political leader's expressive display. Journal of Personality and Social Psychology, 49, 15131529.

Micu, A. C. \& Plummer, J. T. (2010). Measurable emotions: How television ads really work. Journal of Advertising Research, 50, 137-153. doi: 10.2501/S0021849910091300

Moya, S. \& SAlvador, A. (2001). Respuesta cardíaca y electrodérmica ante estresores de laboratorio. Revista Electrónica de Motivación y Emoción, 5, 5-6.

Muñoz, C. \& JimÉnEZ, A. (1990). La expresión de la emoción a través de la conducta social. Revista de Psicología General y Aplicada, 43, 289-299.

Ramos, R. (2006). Contribución al estudio de la calidad de vida y ansiedad en pacientes con hipertensión primaria, con denervación simpática t2-t3. Tesis doctoral no publicada, Universidad de Barcelona, Barcelona

SCHACHTER, S. \& SingER, J. E. (1962). Cognitive social and physiological determinants emotional states. Psychological Review, 69, 379-399. doi: 10.1037/h0046234

Sequeira, H., Hot, P., Silvert, L. \& Delplanque, S. (2009). Electrical autonomic correlates of emotion. International Journal of Psychophysiology, 71, 50-56. doi:10.1016/j.ijpsycho.2008.07.009

Shapiro, D. \& SCHWARTz, G. E. (1970). Psychophysiological contribution to social psychology. Annual Review of Psychology, 21, 87-112.

Sirota, A. D. \& Schwartz, G. E. (1982). Facial muscle patterning and lateralization during elation and depression imagery. Journal of Abnormal Psychology, 91, 25-34. doi: 10.1037/0021-843X.91.1.25

YounG, L. \& SHEENA, D. (1975). Methods and designs: Survey of eye movement recording methods. Behavior Research Methods and Instruments, 7, 397-429.

ZAJONC, R. B. (1965). Social facilitation. Science, 149, 269-274. 